\title{
Extendable nickel complex tapes that reach NIR absorptions
}

\author{
Hassib Audi, ${ }^{a}$ Zhongrui Chen, ${ }^{a}$ Azzam Charaf-Eddin, ${ }^{b}$ Anthony D'Aléo, ${ }^{a}$ Gabriel Canard, ${ }^{a}$ \\ Denis Jacquemin ${ }^{\prime \prime *}$ and Olivier Siri ${ }^{a} * *$
}

${ }^{a}$ CINaM, UMR 7325 CNRS, Aix-Marseille Université, Campus Luminy, case 913, 13288 Marseille Cedex 09, France. E-mail: olivier.siri@univ-amu.fr ${ }^{b}$ CEISAM UMR CNRS 6230, Université de Nantes, 2, rue de la Houssinière - BP 92208 - 4322 NANTES Cedex 3 (F) and Institut Universitaire de France, 103 blvd St Michel, 75005 Paris Cedex 05, France.E-mail: Denis.Jacquemin@univ-nantes.fr

$\dagger \quad$ Electronic Supplementary Information (ESI) available: Experimental details and characterization data of the molecules are described with the spectrophysical studies and quantum mechanical calculations. The X-ray diffraction data of $\mathbf{7}$ have been deposited at the Cambridge Crystallographic Data Centre (CCDC-1011676). See DOI: 10.1039/c000000x/

\section{Stepwise synthesis of linear nickel complex oligomer tapes with no need of solid-phase support has been achieved. The control of the length in flat arrays allows a fine tuning of the absorption properties from the UV to the NIR region.}

Discrete oligomers with a very long $\pi$-conjugated path are of major interest because of both fundamental aspects and applications in a large panel of areas ranging from energy and bio-imaging to sensing and advanced optoelectronics. ${ }^{1-3}$ Numerous attempts have been made to achieve a full control of the $\pi$-electronic length and beside the random and self-organization preparations, the stepwise synthesis of oligomers appears to be the unique method for a full control over the chain length. However, if this elegant method could be successfully applied in organic chemistry to the synthesis of organic oligomers (carbon-carbon bond formation), ${ }^{4-8}$ the access to oligonuclear complexes by coordination chemistry (metal-ligand assembling) encountered dramatic limitations especially for higher oligomers (e.g., synthetic inaccessibility, chemical instability, difficult purification steps and/or poor solubility). As such, these latter could be prepared in a stepwise manner only by solid-assisted syntheses involving electrode, metal surface or insoluble matrix. ${ }^{9-12}$ Among them, the use of resins appears to be the only system which allows reactions of coordination and the release of the final products. ${ }^{9}$

An efficient strategy for maximizing the conjugation is to hold the $\pi$-systems coplanar by assembling repeated metal-ligand units as a tape-like array. In this approach, the ligand design is a crucial factor and previous works reported that low-lying $\pi^{*}$-orbitals of quinoid ligands can mix extensively with the valence $d$-orbitals of a metal center, giving complexes in which the $\pi$-electrons could be fully delocalized over both metal and ligand. ${ }^{13,14}$

Although oxocarbon of type $\mathbf{1}$ is probably the most used quinoid ligand (more than 200 complexes based on mono-, dinuclear compounds, and polymers), ${ }^{15}$ its stepwise metallation leading to complex oligomer tapes has not been described. Surprisingly, the corresponding nitrogenated ligand $\mathbf{2}$ has been much less investigated since only an article of Lever et al. reported its use as ligand (prepared in situ). ${ }^{16}$ The metallation of $\mathbf{2}$ with a ruthenium precursor did not afford the corresponding (and expected) dinuclear complex but instead an oxidized species 3 in which the carbonyl functions impede the communication between the two metal centers. This shortcoming can be explained by the strongly limited access to 2 owing to its in situ preparation in solution, ${ }^{16,17}$ or deposit by sublimation on a CsI plate cooled 
by closed-cycle refrigeration at $15 \mathrm{~K} .{ }^{18}$ In contrast, $\mathrm{N}$-substituted analogues of type $4(\mathrm{R}=$ alkyl or aryl) have attracted much more interest as their easy access paved the way to their use as ligand for the preparation of dinuclear complexes 5 with a high degree of electronic delocalization. ${ }^{19-21}$

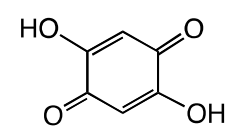

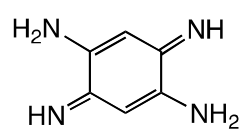

2

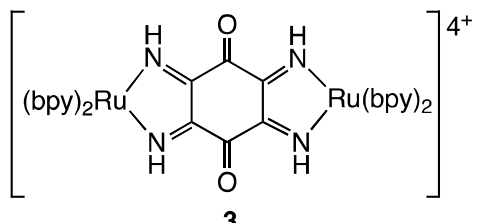

Importantly, no metal complex oligomers based on $\mathbf{4}$ could be attained presumably due to the steric hindrance of the $\mathrm{N}$ - substituents. Consequently, we decided to reinvestigate the isolation of $\mathbf{2}$ to study in detail its coordination chemistry (metal-ligand assembling) since the absence of N-substituents should favour the formation of monodisperse nickel complex tapes with extension of the $\pi$-delocalization.
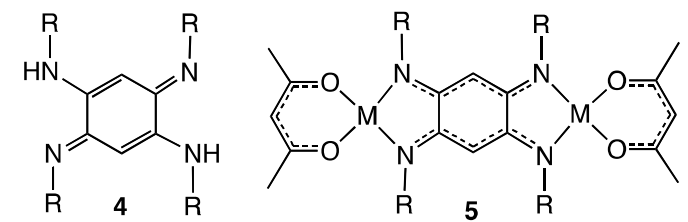

Herein, we report the isolation and full characterization of $\mathbf{2}$ and its use as ligand in the stepwise synthesis of di-, tri-, tetra- and pentanuclear complexes 7-10. The proposed synthetic procedure allows the purification of the oligomers by simple filtration with no need of solid-phase support. Spectro-photophysical and theoretical studies revealed electronic absorption bands in specific regions from UV to NIR depending on the size of the oligomers.

The synthesis of $\mathbf{2}$ was based on the venerable Nietzki's work (1887) ${ }^{17}$ who mentioned in water spontaneous oxidation in air of the electron-rich tetraaminobenzene $\mathbf{6}$ yielding the 2,5-diamino1,4-benzoquinonediimine 2 (Scheme 1). When the same reaction was carried out in methanol, 2 was directly precipitated as a brown solid in 79\% yield, preventing its reaction with 6 and/or other oxidized species. The ${ }^{1} \mathrm{H}$ NMR spectrum of 2 showed only one quinoidal C-H signal at 5.44 ppm because of a fast intramolecular concerted double proton transfer involving two degenerate tautomers which generate in solution an average structure of higher symmetry (see the Supplementary Information, ESI). ${ }^{22}$ The metallation of 2 with [Ni(acac) $)_{2}$ ( 2 equiv.) in toluene at room temperature (Scheme 2) afforded the dinickel complex 7 as a green crystalline solid (54\% yield). When the same reaction was carried out in THF, trinuclear compound $\mathbf{8}$ was precipitated and isolated by simple filtration as a violet solid (77\% yield). Note that $\mathbf{8}$ could be also directly obtained from 7 in THF without any additional reagent. This observation clearly proved that two molecules of 7 evolve in solution into one molecule of 8 and one molecule of [Ni(acac) $)_{2}$. When 8 
was dissolved in DMSO, an unprecedented pentanuclear complex 9 could now be isolated by precipitation ( $89 \%$ yield), its formation occurring via a similar process (two molecules of $\mathbf{8}$ give one of 9 and one of $\left.\left[\mathrm{Ni}(\mathrm{acac})_{2}\right]\right)$. The formation of $\mathbf{9}$ from $\mathbf{2}$ could be also obtained in DMSO confirming the key role of the solvent (i.e. polarity, solubility). Thereby, the oligomerization completely stops when the solubility limit is attained, leading to precipitation of the longest nickel complex from a mixture of oligomers (see ESI). It is noteworthy that a tetranuclear oligomer 10 could be similarly observed (but not isolated) from 7 and 8 (1:1 ratio) in DMSO. Thus, a finetuning of the length becomes possible depending on the nature of the solvent but also of the building blocks.

The ${ }^{1} \mathrm{H}$ NMR spectrum of 7 showed one quinoidal C-H signal at 4.49 ppm consistent with a centrosymmetric molecule that can be explained by a highly delocalized $\pi$-system (see Fig. $1 \mathrm{a}$ and calculations below). ${ }^{19}$ X-ray diffraction study on single crystals of 7 confirmed its centrosymmetry (Fig. 2). The dianion derived from $\mathbf{2}$ acts as a tetradentate bridging ligand in a bis(chelating) fashion and the geometry around the metals is square planar with the nickel being quasi in the molecular plane of 7 (mean plane deviation of 0.059 and $0.002 \AA$ for $\mathrm{Ni}(2)$ and $\mathrm{Ni}(1)$, respectively). The $\mathrm{Ni} \cdot \mathrm{Ni}$ separation of $7.65 \AA$ is in the line of that found in similar dinuclear nickel complex containing ligand of type 2. ${ }^{19-21,23}$ Examination of the bond distances within the $\mathrm{N}(1)-\mathrm{C}(1)-\mathrm{C}(2)-\mathrm{C}(3)-\mathrm{N}(3)$ and $\mathrm{N}(2)-\mathrm{C}(6)-\mathrm{C}(5)-\mathrm{C}(4)-\mathrm{N}(4)$ moieties is consistent with an extensive $\pi$-electronic delocalization, but with no significant conjugation between the two $6 \pi$-subsystems since the C(1)-C(6) and C(3)-C(4) distances are typical of single bonds (1.498(3) and 1.494(3) $\AA$, respectively). ${ }^{9}$

Molecule $\mathbf{8}$ was experimentally characterized by NMR spectroscopy and high-resolution mass spectrometry (HRMS) (see the ESI).

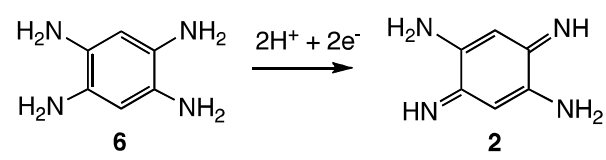

Scheme 1. Synthesis of aminobenzoquinonediimine 2. 

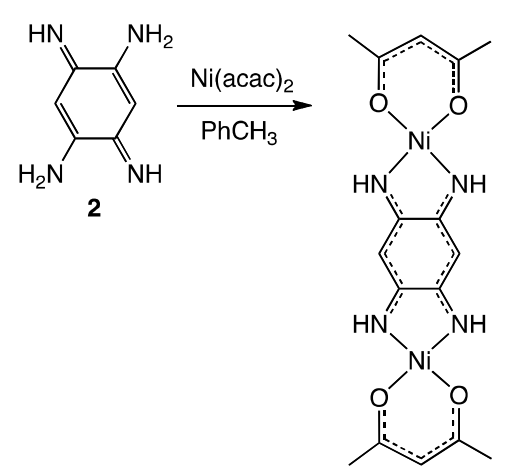

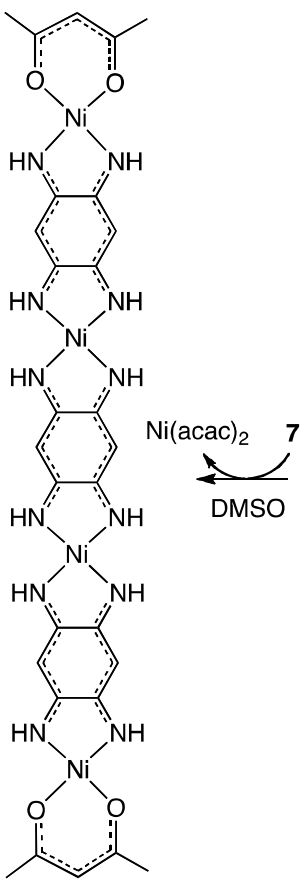

10

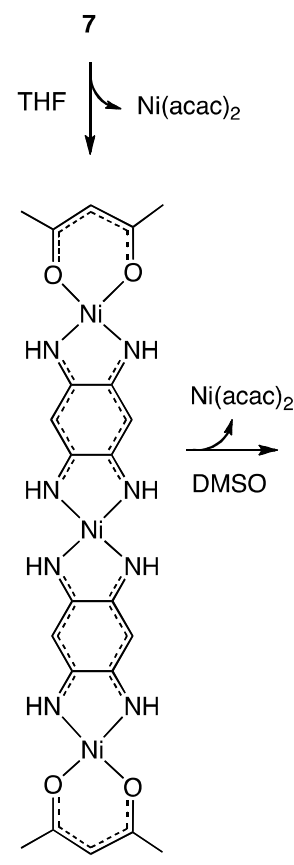

8

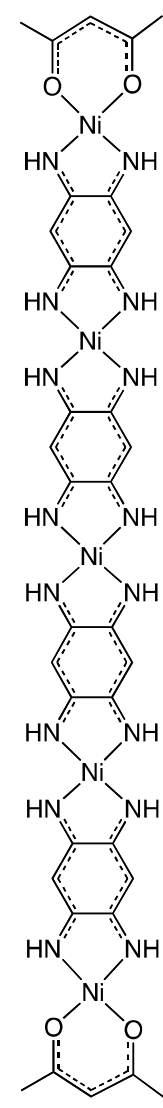

9

Scheme 2. Synthesis of oligomers 7-10.

The HRMS spectrum of 8 shows a peak at 641.0213 mass/charge ratio $(\mathrm{m} / \mathrm{z})\left[(\mathrm{M}+\mathrm{H})^{+}\right]$as expected for $\mathrm{C}_{22} \mathrm{H}_{22} \mathrm{~N}_{8} \mathrm{O}_{4} \mathrm{Ni}_{3}$. Its ${ }^{1} \mathrm{H}$ NMR spectrum showed one olefinic $\mathrm{C}-\mathrm{H}$ signal at $4.63 \mathrm{ppm}$ integrating for 4 protons (the two acac $\mathrm{C}-\mathrm{H}$ protons are used as standard reference since $\mathrm{I}=2 \mathrm{in}$ all cases, see Fig. 1b). The formation of the tetranuclear complex $\mathbf{1 0}$ from $\mathbf{7}$ and $\mathbf{8}$ in solution could be followed by ${ }^{1} \mathrm{H}$ NMR spectrum (see the ESI) that revealed the presence of a broad signal around $4.75 \mathrm{ppm}$ integrating for $6 \mathrm{C}-\mathrm{H}$ quinoidal protons $(\mathrm{I}=6 \mathrm{H})$. Unfortunately, its isolation as a solid could not be achieved because of its rapid co-precipitation with 9 that is also formed during the reaction between 7 and $\mathbf{8}$ (1:1 ratio) in DMSO. In contrast, pentanuclear complex 9 could be isolated (directly from 8) by precipitation in DMSO as a blue solid. Its characterization could be carried out only in solid state (elemental analysis and solid NMR using 8 as reference, see ESI) owing to its very poor solubility.

Ligand 2 shows an intense absorption band at $317 \mathrm{~nm}$ (Fig. 3) that is assigned to the intraquinone transitions. ${ }^{13}$ Its stepwise metallation could be followed in DMSO by UV-Vis-NIR 
absorption, indicating the successive $\mathbf{7} \rightarrow \mathbf{8} \rightarrow \mathbf{9}$ and/or $\mathbf{1 0}$ conversion (see ESI). The absorption spectra of 7 and 8 exhibit broad bands peaking at 576 and $627 \mathrm{~nm}$, respectively (Fig. 3).
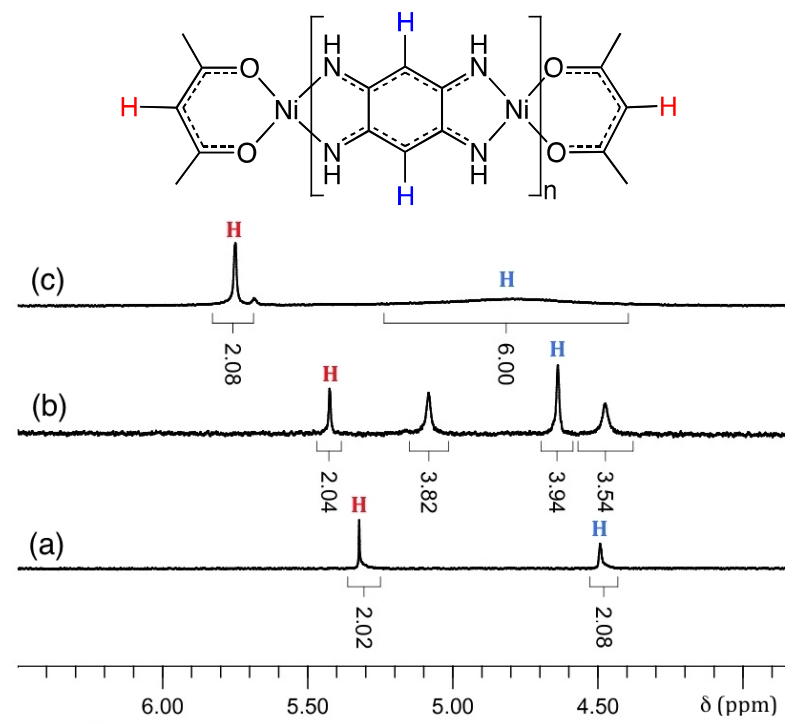

Fig. $1{ }^{1} \mathrm{H}$ NMR spectrum of: (a) $7(n=1)$ in $\mathrm{CDCl}_{3}$, (b) $8(n=2)$ and (c) $10(n=3)$ in DMSO- $d_{6}$. The range $4<\delta<0$ ppm has been omitted for clarity. Colour code : red (acac C-H proton), blue (quinoidal C-H proton).

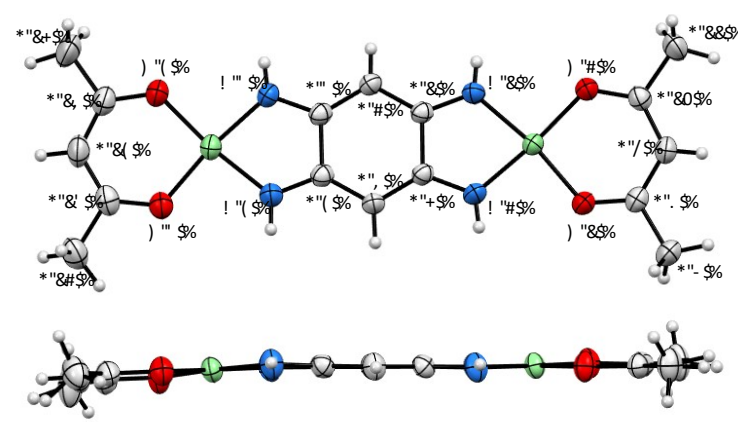

Fig. 2 Structure of 7 (anisotropic displacement parameters at 50\%) : top view and side view. Selected bond lengths $(\AA)$ : $N(4)-C(4)=1.319(3), C(4)-C(5)=1.394(3), C(5)-C(6)=1.393(3), C(6)$ $\mathrm{N}(2)=1.320(3), \mathrm{N}(1)-\mathrm{C}(1)=1.318(3), \mathrm{C}(1)-\mathrm{C}(2)=1.379(3), \mathrm{C}(2)-\mathrm{C}(3)=1.386(3), \mathrm{C}(3)-\mathrm{N}(3)=$ $1.316(3), C(1)-C(6)=1.498(3), C(3)-C(4)=1.494(3)$. 


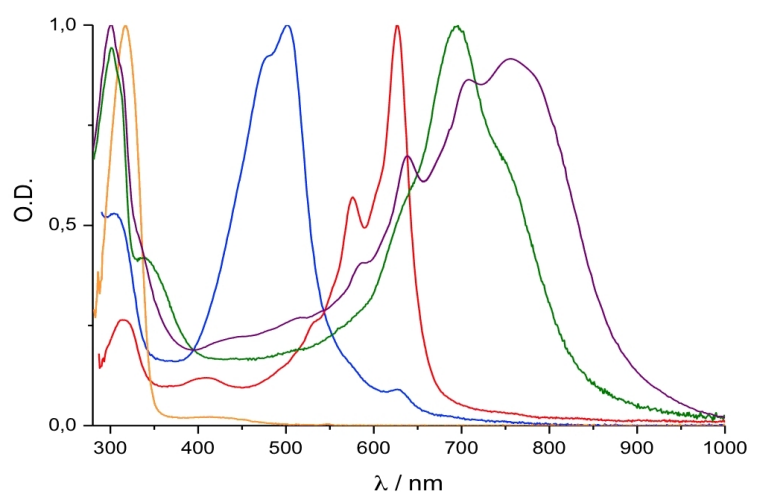

Fig. 3 UV-vis-NIR absorption spectrum of $\mathbf{2}$ and its oligomers. Colour code: $\mathbf{2}$ in $\mathrm{CH}_{2} \mathrm{Cl}_{2}$ (orange), 7 in $\mathrm{CH}_{2} \mathrm{Cl}_{2}$ (blue), 8 in THF (red), 10 in DMSO (green)* and 9 in DMSO (violet)*. (*) derived from a mixture in solution.

The pronounced red shift of the absorption of $\mathbf{8}$ with respect to that of 7 results from an extension of the delocalization of the conjugated $\pi$-system. The electronic absorption spectra of the trimer 10 and of the tetramer 9 peaking at $\lambda_{\mathrm{abs}}=693$ and $757 \mathrm{~nm}$, respectively, confirmed this hypothesis. As expected, extension of the tape length increases the bathromic shift, allowing a fine-tuning of the absorption in the entire visible region as well as in the NIR region.

In order to gain more insights into the relationship between the electronic spectrum and the $\pi$ electron delocalization in these compounds, theoretical calculations were performed on 7-10 (see the ESI for details). These studies show that the optimal structures are either perfectly planar (7), or very close to planarity. For instance, the longest tape 9 belongs to the $D_{2}$ point group, but the deviation from planarity is small suggesting that crystal packing effects might yield perfectly flat compounds (Fig. 4). The DFT-computed HOMO-LUMO gap (HLG) for 7, 8, 10, 9 are 3.22, 2.81, 2.63 and $2.55 \mathrm{eV}$, confirming the constant decay of the gap with increasing size (see ESI). More impressive is the increase of the isotropic polarisability that values 460, 807, 1179 and 1558 a.u. for the same four compounds, respectively, highlighting the strong increase of $\pi$-delocalization. The TD-DFT calculations on the solvated molecules reveal for all one strongly dipole-allowed $\mathrm{S}_{0^{-}}$ $\mathrm{S}_{1}$ transition in the visible domain. The delta density plots can be found in Fig. 5 for the four members of the series. The delta density plots can be found in Fig. 5 for the four members of the series. It is obvious that the excited state correspond to a typical $\pi-\pi *$ transition with alternating regions of gain/depletion of density, the single-bonds separating the two sub-structures (e.g., $\mathrm{C}(4)-\mathrm{C}(3)$ and $\mathrm{C}(1)-\mathrm{C}(6)$ in 7) gaining electron density (mostly in red) after absorption, as expected. One notices that the excited-state spreads over several units, so that one needs to reach rather long tapes to obtain convergence of the transition energies. TD-DFT calculations performed for 7, 8, 10,9 as well as the longer hexanuclear and heptanuclear compounds provide vertical transition wavelengths at 426,512,581, 605, 623 and $643 \mathrm{~nm}$, respectively. 


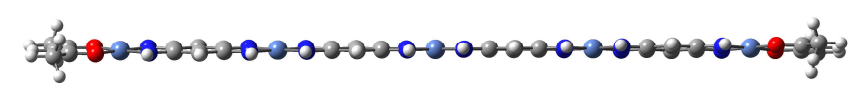

Fig. 4 DFT optimal structure of the pentanuclear complex 9

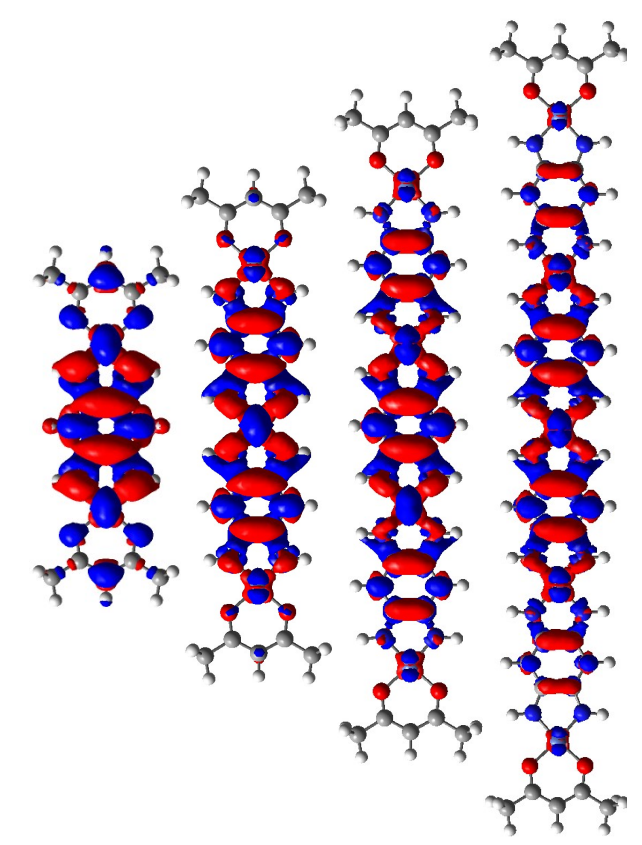

Fig. 5 M06/6-31G(d) Delta density plots between the excited and ground states for the 7, 8, 10 and 9 systems. Red (blue) regions indicate increase (decrease) of the density upon absorption. The selected contour threshold is 0.0004 a.u.

Though all values are blue-shifted compared to the experiment, the evolution with oligomeric length is valid, e.g., $-1.00 \mathrm{eV}$ (theory) and $-0.83 \mathrm{eV}$ (experiment) when going from 7 to 9 and indicate that further bathochromic shifts could be reached with longer tapes.

In summary, we report the rapid, easy and stepwise synthesis of mono- di- tri- and tetramer 710 with extension of the $\pi$-delocalization. The synthetic accessibility - based on the unique behaviour of ligand $\mathbf{2}$ and the key role of the solvent (oligomerization completely stops when the solubility limit is attained) - is highly efficient because of a purification step by simple filtration with no need of solid-phase support. Importantly, the possibility of controlling the length of the oligomers allows a fine tuning of the absorption properties from the UV to the NIR region. This unique example upon stepwise metallation opens diverse and extensive perspectives in many technological sectors. ${ }^{1}$ Last but not least, the combination of both redox properties of the transition metals (Ni) and ligands (2) in 7-10 is considered to provide multiredox systems for functionalized materials and catalysts. ${ }^{24-29}$

OS acknowledges the Centre National de la Recherche Scientifique, the Ministère de la Recherche et des Nouvelles Technologies for financial support and $\mathrm{PhD}$ grant of ZC. DJ acknowledges the European Research Council (ERC) and the Région des Pays de la Loire for 
financial support in the framework of a Starting Grant (Marches - 278845) and a recrutement sur poste stratégique, respectively. This research used resources of 1) the GENCI-CINES/IDRIS (Grants c2012085117), 2) CCIPL (Centre de Calcul Intensif des Pays de Loire), 3) the local Troy cluster acquired thanks to Région des Pays de la Loire.

\section{Notes and references}

1 G. Qian, Z. Y. Wang, Chem. Asian J. 2010, 5, 1006.

2 H. Jiang, Macromol. Rapid Commun. 2010, 31, 2007.

3 Electronic Materials: The Oligomer Approach (Eds.: K. Müllen, G. Wegner), Wiley-VCH, Weinheim, 1998.

4 L. Chen, C. Li, K. Müllen, J. Mater. Chem. C, 2014, 2, 1938.

5 Z. Zeng, S. Lee, J. L. Zafra, M. Ishida, X. Zhu, Z. Sun, Y. Ni, R. D. Webster, R.-W. Li, J. T. Lòpez Navarrete, C. Chi, J. Ding, J. Casado, D. Kim, J. Wu, Angew. Chem. Int. Ed. 2013, 52, 8561.

6 Y. Li, J. Gao, S. Di Motta, F. Negri, Z. Wang, J. Am. Chem. Soc. 2010, 132, 4208.

7 T. Hori, X. Peng, N. Aratani, Takagi, T. Matsumoto, T. Kawai, Z. S. Yoon, M.-C. Yoon, J. Yang, D. Kim, A. Osuka Chem. Eur. J. 2008, 14, 582.

8 J. He, J. L. Crase, S. H. Wadumethrige, K. Thakur, L. Dai, S. Zou, R. Rathore, S. Hartley J. Am. Chem. Soc. 2010, 132, 13848.

9 K. Heinze, J. D. Bueno Toro, Angew. Chem. Int. Ed. 2003, 42, 4533.

10 H. Maeda, R. Sakamoto, Y. Nishimori, J. Sendo, F. Toshimitsu, Y. Yamanoi, H. Nishihara Chem. Commun. 2011, 47, 8644.

11 H. Nishihara, K. Kanaizuka, Y. Nishimori, Y. Yamanoi Coord. Chem. Rev. 2007, 251, 2674.

12 H. Maeda, R. Sakamoto, H. Nishihara Polymer 2013, 54, 4383.

13 H. Masui, A. B. P. Lever, Inorg. Chem. 1993, 32, 2199.

14 R. A. Metcalfe, L. C. G. Vasconcellos, H. Mirza, D. W. Franco, A. B. P. Lever, J. Chem. Soc., Dalton Trans. 1999, 2653.

15 S. Kitagawa, S. Kawata, Coord. Chem. Rev. 2002, 224, 11.

16 H. Masui, A. L. Freda, M. C. Zerner, A. B. P. Lever, Inorg. Chem. 2000, 39, 141.

17 R. Nietzki, E. Hagenbach, Ber. Dtsch. Chem. Ges. 1887, 20, 328; ibidem 2114.

18 K. Ujike, S. Kudoh, M. Nakata, Chem. Phys. Lett. 2005, 409, 52.

19 O. Siri, J.-p. Taquet, J.-P. Collin, M. D. Rohmer, M. Bénard, P. Braunstein Chem. Eur. J. 2005, 11, 7247.

20 O. Siri, P. Braunstein, J.P. Taquet, J.P. Collin, R. Welter, Dalton Trans. 2007, 1481.

21 J.-p. Taquet, O. Siri, P. Braunstein, R. Welter Inorg. Chem., 2006, 45, 4668.

22 H. Rumpel, H. H. Limbach, J. Am. Chem. Soc. 1989, 111, 5429.

23 C. G. Pierpont, L. C. Francesconi, D. Hendrickson, Inorg. Chem. 1977, 16, 2367.

24 T.Moriuchi, T. Hirao, Acc. Chem. Res. 2012, 45, 347.

25 Y.Ishii, S. Sakaguchi,; T. Iwahama, Adv. Synth. Catal. 2001, 343, 393.

26 M. D. Ward, J. A. McCleverty J. Chem. Soc., Dalton Trans. 2002, 275.

27 J.-E. Bäckvall, Modern Oxidation Methods; Wiley-VCH:Weinheim, Germany, 2004.

28 B. J. Holliday, T. M. Swager, Chem. Commun. 2005, 23.

29 H. Nishihara, Coord. Chem. Rev. 2005, 249, 1468.

30 M. O. Wolf, J. Inorg. Organomet. Polym. Mater. 2006, 16, 189. 